\title{
Network analysis of heart beat intervals using horizontal visibility graphs
}

\author{
Tamas Madl ${ }^{1,2}$ \\ ${ }^{1}$ Austrian Research Institute for Artificial Intelligence ${ }^{2}$ University of Manchester
}

\begin{abstract}
Heart beat interval time series contain information predictive of heart disease, but most current predictors do not provide sufficient reliability for clinical use. Using several predictors improves predictive power, but the limit is not yet known, suggesting that not all the information in interbeat interval series has been captured by previous work.

We convert heart beat time series into scale-free networks using horizontal visibility graphs (HVGs), which are well-suited to distinguishing deterministic dynamical systems from stochastic systems, allowing them to model new aspects of autonomic heart rate modulation. Based on the $H V G$, we introduce and evaluate a general class of predictors, which can be used to augment existing features used in heart rate variability $(H R V)$ analysis, and which exhibit high predictive power for several types of heart disease.

We show the statistical significance of these network predictors, and their competitive performance to popular statistical, geometric and non-linear features, on ICU and Holter ECGs, including several heart disease etiologies.
\end{abstract}

\section{Introduction}

Heart rate variability (HRV) analysis is based on time series of intervals between heart beats (R-R intervals), which have been argued to contain information predictive of some types of heart disease, such as heart failure. Low heart rate variability has been proposed to indicate reduced cardiac regulatory capacity and is a strong predictor of mortality and health issues [1-3].

However, current HRV analysis methods are not reliable enough for widespread clinical practice [4]. No single predictor is powerful enough to facilitate high prognostic power. For this reason, several authors have argued for a combination of several different predictors $[5,6]$; but the limits of this approach have not yet been reached [5], suggesting that there is under-utilized information.

Several families of predictors have been used, including statistical features, geometric features (based on empirical sample density distributions of the R-R intervals), nonlinear features (based on tools from non-linear dynamical systems theory to infer and characterize system behavior, such as attractor reconstruction), and frequency-domain methods (separating and analysing spectral components at different frequencies) [4]. We describe and evaluate some representative examples in Sections 2 and 3 below.

Here, we propose a new class of predictors based on complex networks. The key idea is the conversion of the time series into a graph, while preserving its structure and important properties, in order to facilitate the use of complex network analysis tools.

\subsection{Horizontal Visibility Graphs}

A horizontal visibility graph is a network constructed of a time series $\left(t_{1}, x_{1}\right), \ldots,\left(t_{n}, x_{n}\right)$, such that each $x$ has a corresponding vertex, each pair of vertices corresponding to a pair of values $x_{a}$ and $x_{b}$ is connected by an edge if both $x_{a}, x_{b}>x_{n}$ for all $a<n<b$ (see Figure 1). HVGs have been argued to be well-suited to distinguishing stochasticity from deterministic dynamical systems [7], and thus are useful for HRV analysis (based on the argument of autonomic modulation influencing variability).

The HVG of a time series is invariant under affine transformations, preserves structural properties (such as periodicity and fractality, shown to be highly relevant for HRV analysis [8]), and can discriminate stochastic and chaotic processes [7]. In addition to these properties, the conversion to a graph opens the door to performing complex network analysis on R-R time series [9].

\subsection{Possible Causes of HRV and Corre- sponding Complex Network Features}

Several explanations for the impact of disease on HRV have been proposed (see $[3,10]$ for reviews). For example, [11] implicated the effect of vagal tone on beat to beat fluctuation (a rapidly reacting sympathetic control system adjusting heart rhythm upon beat-to-beat perturbations due to e.g. blood pressure changes or movements). In contrast, [12] suggested fractal properties of normal heart rhythms to arise from nonlinear regulatory systems, and hypothesized their break down with reduced adaptive capacity.

These hypothesized mechanisms implicate some network descriptors to be more plausibly useful than others. 


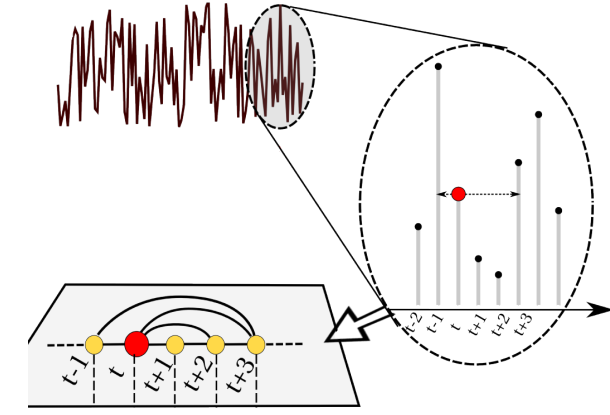

Figure 1. Horizontal visibility graph analysis. From a time series of heart beat intervals (top), those pairs of values which are 'visible' - such that they are greater than all values in between (right) - are connected via an undirected edge, yielding a graph representation (bottom) which retains key properties of the structure of the input time series (e.g., periodicity and fractality)

The effects of a sympathetic control system decreasing variability by over-regulating perturbations [11] should be visible using basic graph properties such as its diameter (maximum of all eccentricities) or radius (minimum of eccentricities), where eccentricity of $x_{a}$ is defined as the distance to the vertex $x_{b}$ farthest from it. Finally, the connectivity of the graph can be quantified using its transitivity (fraction of all possible triangles present in the graph).

The suitability of these features to model variability directly follows from the the relationship between visibility and variability [7]. Briefly, a time series without variability would lead to no visibility beyond adjacent neighbors, and thus to a linear chain (with very large radius / diameter and few central nodes), whereas maximal variability would yield strongly connected graphs with opposite properties.

On the other hand, [12] suggests fractal properties, which are frequently exhibited by small-world networks [13], indicating that the 'small-world-ness' of a network may be a good metric for indirectly modelling the activity of the postulated nonlinear regulatory system using R-R time series. Small world networks allow reaching any vertex from any other vertex using a very small number of steps. The clustering coefficient [14] can be used to determine to what extent a graph is a small world network.

The average shortest path length and the degree assortativity (a measure of connectivity of vertices with the same degree - usually there are large differences in small world network due to clustering) are measuring related properties. In addition to assortativity, which has been studied before, we also propose a novel connectivity measure, disassortative entropy, defined as the entropy of the mixing matrix (which contains the joint probabilities of edges of the same degree - see Section 2.2) which measures the expected information content of the tendency of vertices to connect to similar vertices. A graph always connecting high-degree vertices to other high-degree vertices would maximize assortativity, a graph doing the oppositive would minimize it, and a graph in which the connectivity is randomized would maximize disassortative entropy (thus, this feature is a second-degree measure of stochasticity).
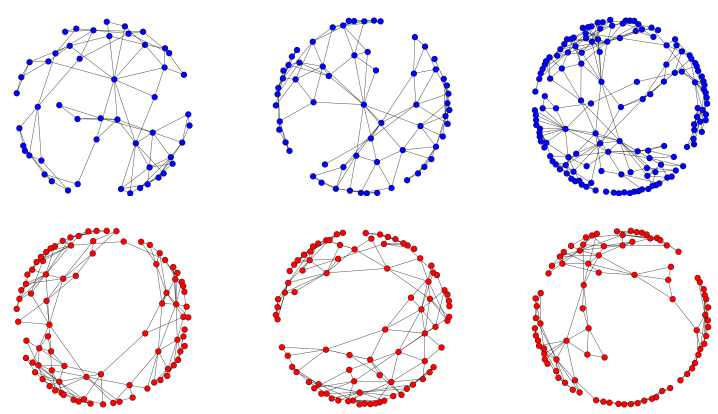

Figure 2. Horizontal visibility graphs of R-R series extracted from ECGs of three healthy participants (top row) and three patients with congestive heart failure, chronic pulmonary heart disease, and primary cardiomyopathy respectively (bottom row). Note the higher centrality, heavier connectivity, and clustering in the top row, and the tendency to build a simple ring (corresponding to small amounts of variability and fractality) in the bottom row

\section{Methods}

Two datasets were used as the data source for patients with heart disease, the Multi-Parameter Intelligent Monitoring for Intensive Care (MIMIC) II dataset [15] and the Coronary Artery Disease (CAD) Holter data from the Telemetric and Holter ECG Warehouse [16]. We extracted the subset of MIMIC II that 1) contained ECG waveforms matched to ICD-9 codes and 2) corresponded to one of the four disease etiologies modelled below - congestive heart failure $(\mathrm{CHF})$, primary cardiomyopathy (PCM), and chronic pulmonary heart disease (CPHD) and CAD. From each subject's corresponding waveforms, up to $10 \mathrm{R}-\mathrm{R}$ time series, each with one minute duration, were extracted using the most recent version of PhysioNet's ecgpuwave beat detector [17]. In total, there were 613 congestive heart failure, 74 cardiomyopathy, and 71 chronic pulmonary disease patients in MIMIC II, and 271 patients in the CAD data set.

For verified healthy control data, we have used the MITBIH Normal Sinus Rhythm database (18 participants aged 26 to 50, including 5 men and 13 women) and the PhysioNet Fantasia dataset (twenty young - 21-34 years old and twenty elderly - 68-85 years old - rigorously-screened healthy subjects), a total of 58 healthy control participants; once again extracting 10 one-minute R-R time series. 
To test whether the suggested features are predictive, for each of the disease etiologies mentioned above, we perform a non-parametric Mann-Whitney U test [18]. We also compared predictive power, reporting areas under the Receiver Operating Characteristic (ROC) curve (AUC) [19] with and without adding proposed network features to a recent HRV classification system [5] based on a random forest with 30 trees [20], and five-fold cross-validation.

\subsection{Network features}

We calculated the following features (see Section 1). Let $G$ denote the graph, lower-case characters vertices and $V_{G}$ the set of all vertices in $G$, and $d(a, b)$ the distance between two vertices (defined as the shortest walk along the existing edges). Furthermore, let $\operatorname{deg}(v)$ be the number of edges connected to vertex $v$, and $\operatorname{ecc}(v)=\max _{x \in V_{G}}\{d(v, x)\}$ be the eccentricity of a vertex $v$.

1. Diameter: $\operatorname{diam}(G)=\max _{x \in V_{G}}\{\operatorname{ecc}(x)\}$

2. Radius: $\operatorname{rad}(G)=\min _{x \in V_{G}}\{\operatorname{ecc}(x)\}$

3. Transitivity: $T(G)=|\operatorname{Tr} i(G)| /|\operatorname{Tr} i(V)|$, where $\operatorname{Tri}(G)$ is the set of all triangles in $G$, and $\operatorname{Tri}(V)$ the set of all possible triangles given all vertices $V$

4. Clust. coefficient: $C(G)=\frac{1}{\left|V_{G}\right|} \sum_{v \in G} \frac{2|\operatorname{Tr} i(v)|}{\operatorname{deg}(v)(\operatorname{deg}(v)-1)}$, where $\operatorname{Tri}(v)$ is the set of all triangles through vertex $v$

5. Avg. shortest path length: $l(G)=\sum_{a, b \in V_{G}} \frac{d(a, b)}{\left|V_{G}\right|\left(\left|V_{G}\right|-1\right)}$ 6. Assortativity: $r(G)=\left(\sigma_{a} \sigma_{b}\right)^{-1} \sum_{x y} x y\left(e_{x y}-a_{x} b_{y}\right)$, where $e_{x y}$ is the joint probability of degrees of vertices $x$ and $y$, and $a_{x}$ and $b_{y}$ are the fraction of edges starting / ending at vertices $x$ and $y$. See [21] for details.

7. Disassortative entropy: $E(G)=\sum_{y} \sum_{x} e_{x y} \log e_{x y}$

\subsection{Comparison features}

We also calculate the following, previously proposed statistical, geometric and non-linear features of a time series $S=\left(x_{1}, \ldots, x_{n}\right)$ of interbeat intervals for comparison. We closely follow the setup of [5], in order to facilitate quantitative comparison with a state of the art system.

Statistical:

8. Standard deviation: $S D N N(S)=\sigma(S)=\sqrt{\operatorname{Var}[S]}$

9. Root mean squared standard deviation: $R M S S D=$ $|S|^{-1} \sqrt{\sum_{i=1}^{|S|-1}\left(x_{i+1}-x_{i}\right)^{2}}$

10. Ratio of the number of successive R-R interval differences greater than $20 \mathrm{~ms}$ to the total number of intervals: $p N N 20=\left|x_{i}:\left(x_{i+1}-x_{i}\right)>20\right| /|S|$

11. - 14. Approximate entropy $\operatorname{ApEn}(m, r,|S|)=$ $\Phi(r)-\Phi^{2}(r)$, calculated for four different values $r \in$ $0.1 \sigma(S), 0.15 \sigma(S), 0.2 \sigma(S), 0.25 \sigma(S)$. See [5].

15. Geometric: HRV triangular index: $H R I=|S| / N$. $N$ is the no. of intervals in the modal bin of a histogram

Dynamical systems (each of these features is calculated five times, at delay parameters $T \in\{1,2,5,10,20\}$ [5]):
16. - 20. Spatial Filling Index: $S F I=s / n^{2}$, where $\mathrm{s}$ is a combined factor of the point distribution in phase space (calculated using time-delay embedding in 2D) and $n$ is the number of squares used to estimate the distribution [5] 21. - 25. Central Tendency Measure: $C T M=$ $\sum_{i=1}^{|S|-2} \delta\left(\Delta_{i}\right)$, where $\delta\left(\Delta_{i}\right)=1$ if and only if $\sqrt{\left(x_{i+2}-x_{i+1}\right)^{2}+\left(x_{i+1}-x_{i}\right)^{2}}<r$ and 0 otherwise. 26. - 30. Correlation Dimension of the 2D embedding: $D_{2}=\lim _{\epsilon \epsilon^{\prime} \rightarrow 0^{+}}\left(\ln \left(\epsilon / \epsilon^{\prime}\right)\right)^{-1} \log \left(C(\epsilon) / C\left(\epsilon^{\prime}\right)\right)$, where $C$ is the correlation integral (see [5] for details).

\section{Results}

We evaluated the proposed features on three heart diseases from MIMIC II, and on the CAD dataset. p-values of Mann-Whitney U tests of the positive vs. negative group are $P<<0.001$ for all seven network features and all disease types. The network based features are not strongly correlated to the comparison features (average correlation between network and all other features on all disease types: $r=0.006$ ), suggesting that network features capture different information from existing features.

Figure 3 compares accuracy and AUC scores across features. Network features (1-7) achieve over $80 \%$ accuracy on their own, with the HVG diameter achieving $90 \%$. The AUC scores achieved by the best two single network predictors $\left(A U C_{\text {diameter }}=0.730, A U C_{\text {radius }}=0.730\right)$ are significantly higher than that of the best comparison predictor $\left(A U C_{C T M}=0.681\right)$, with p-values of 0.021 and 0.022 according to Mann-Whitney U tests. The first three features, inspired by the hypothesis of sympathetic overregulation, outperform the others, which partially capture fractal and connectivity structure. Within the connectivity features, the completely novel predictor disassortive entropy ( $A U C=0.659$ ) shows a significant improvement over both assortativity ( $A U C=0.586$ ) and clustering ( $A U C=0.566)$, which have been explored before [22].

We have also implemented a state of the art multifeature HRV classification system [5], and compared it with and without network features on MIMIC II (Table 1).

Table 1. Classification performance (accuracy [acc] / AUC) without and with network features - comparison

\begin{tabular}{lccc}
\hline CVD & Score w/o. & Score w. & p-val. \\
\hline \hline PCM acc & $0.851 \pm 0.023$ & $0.902 \pm 0.023$ & $0.036^{*}$ \\
PCM AUC & $0.926 \pm 0.017$ & $0.960 \pm 0.010$ & $0.021^{*}$ \\
\hline CPHD acc & $0.848 \pm 0.019$ & $0.904 \pm 0.027$ & $0.036^{*}$ \\
CPHD AUC & $0.927 \pm 0.020$ & $0.961 \pm 0.014$ & $0.037^{*}$ \\
\hline CHF acc & $0.938 \pm 0.008$ & $0.948 \pm 0.009$ & 0.210 \\
CHF AUC & $0.921 \pm 0.032$ & $0.947 \pm 0.025$ & 0.140 \\
\hline CAD acc & $0.736 \pm 0.002$ & $0.745 \pm 0.001$ & $0.011^{*}$ \\
CAD AUC & $0.801 \pm 0.003$ & $0.811 \pm 0.002$ & $0.030^{*}$ \\
\hline
\end{tabular}




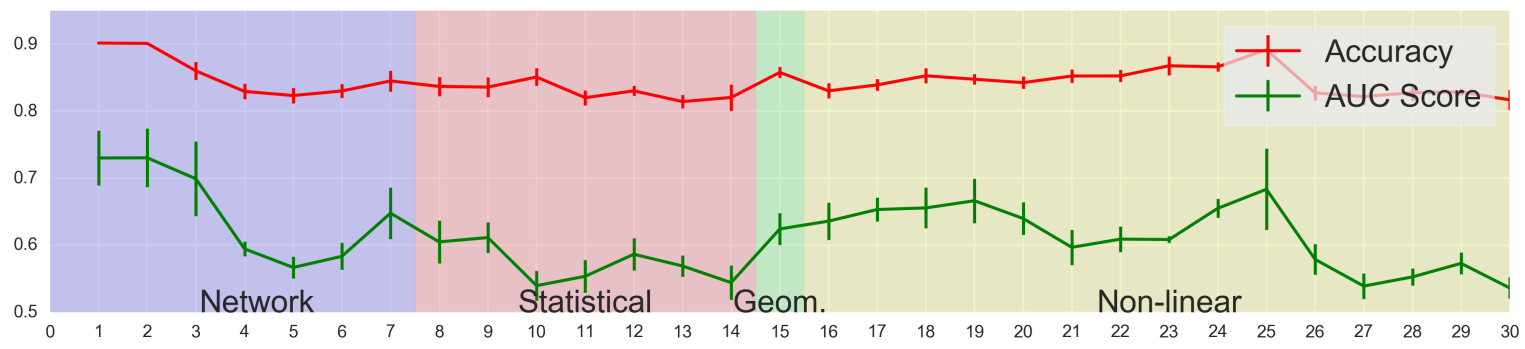

Figure 3. Predictive power (accuracy and AUC) of single network features (1-7) and single comparison features (8-30)

\section{Conclusion}

We introduced and evaluated a class of descriptors for HRV analysis, based on HVGs. The observations that they are decorrelated from traditional HRV features, and can significantly outperform them, suggests that it may be worthwhile to augment HRV analyses with network predictors, and to investigate HVG predictors in future work.

\section{Acknowledgements}

The Telemetric and Holter ECG Warehouse of the University of Rochester and by PhysioNet provided the data.

\section{References}

[1] Tsuji H, Venditti F, Manders ES, Evans JC, Larson MG, Feldman CL, Levy D. Reduced heart rate variability and mortality risk in an elderly cohort. the framingham heart study. Circulation 1994;90(2):878-883.

[2] Dekker JM, Schouten EG, Klootwijk P, Pool J, Swenne CA, Kromhout D. Heart rate variability from short electrocardiographic recordings predicts mortality from all causes in middle-aged and elderly men the zutphen study. American Journal of Epidemiology 1997;145(10):899-908.

[3] Shaffer F, McCraty R, Zerr CL. A healthy heart is not a metronome: an integrative review of the heart's anatomy and heart rate variability. Frontiers in psychology 2014;5.

[4] Xhyheri B, Manfrini O, Mazzolini M, Pizzi C, Bugiardini R. Heart rate variability today. Progress in cardiovascular diseases 2012;55(3):321-331.

[5] Jovic A, Bogunovic N. Electrocardiogram analysis using a combination of statistical, geometric, and nonlinear heart rate variability features. Artificial intelligence in medicine 2011;51(3):175-186.

[6] Wan-tai M, Reinhall PG, Poole JE, Anderson J, Johnson G, Fletcher RD, Moore HJ, Mark DB, Lee KL, Bardy GH. Scd-heft: Use of rr interval statistics for long-term risk stratification for arrhythmic sudden cardiac death. Heart Rhythm 2015;12(10):2058-2066.

[7] Luque B, Lacasa L, Ballesteros F, Luque J. Horizontal visibility graphs: Exact results for random time series. Physical Review E 2009;80(4):046103.

[8] Tapanainen JM, Thomsen PEB, Køber L, Torp-Pedersen C,
Mäkikallio TH, Still AM, Lindgren KS, Huikuri HV. Fractal analysis of heart rate variability and mortality after an acute myocardial infarction. The American journal of cardiology 2002;90(4):347-352.

[9] Albert R, Barabási AL. Statistical mechanics of complex networks. Reviews of modern physics 2002;74(1):47.

[10] E Papaioannou V, O Verkerk A, S Amin A, MT de Bakker J. Intracardiac origin of heart rate variability, pacemaker funny current and their possible association with critical illness. Current cardiology reviews 2013;9(1):82-96.

[11] Akselrod S, Gordon D, Ubel FA, Shannon DC, Berger A, Cohen RJ. Power spectrum analysis of heart rate fluctuation: a quantitative probe of beat-to-beat cardiovascular control. science 1981;213(4504):220-222.

[12] Goldberger AL, Amaral LA, Hausdorff JM, Ivanov PC, Peng CK, Stanley HE. Fractal dynamics in physiology: alterations with disease and aging. Proceedings of the National Academy of Sciences 2002;99(suppl 1):2466-2472.

[13] Rozenfeld HD, Song C, Makse HA. Small-world to fractal transition in complex networks: a renormalization group approach. Physical review letters 2010;104(2):025701.

[14] Watts DJ, Strogatz SH. Collective dynamics of smallworldnetworks. nature 1998;393(6684):440-442.

[15] Saeed M, Lieu C, Raber G, Mark RG. Mimic ii: a massive temporal icu patient database to support research in intelligent patient monitoring. In Computers in Cardiology, 2002. IEEE, 2002; 641-644.

[16] Couderc JP. The telemetric and holter ecg warehouse (thew): the first three years of development and research. Journal of electrocardiology 2012;45(6):677-683.

[17] Jane R, Blasi A, García J, Laguna P. Evaluation of an automatic threshold based detector of waveform limits in holter ecg with the qt database. In Computers in Cardiology 1997. IEEE, 1997; 295-298.

[18] Mann HB, Whitney DR. On a test of whether one of two random variables is stochastically larger than the other. The annals of mathematical statistics 1947;50-60.

[19] Obuchowski NA. Roc analysis. American Journal of Roentgenology 2005;184(2):364-372.

[20] Breiman L. Random forests. Machine learning 2001; 45(1):5-32.

[21] Newman ME. Mixing patterns in networks. Physical Review E 2003;67(2):026126.

[22] Shao ZG. Network analysis of human heartbeat dynamics. Applied Physics Letters 2010;96(7):073703. 